\title{
CDNA Array
}

National Cancer Institute

\section{Source}

National Cancer Institute. cDNA Array. NCI Thesaurus. Code C19819.

A molecular biology device that utilizes a set of defined cDNA clones attached in a specific grid arrangement to a solid support for nucleic acid hybridization assays in gene mapping studies or in determining gene sequences, sequence variations, or gene expression patterns. 